\title{
LOS HILOS LARGOS DE LA TRAMA: APUNTES ETNOGRÁFICOS Y ANÁLISIS DE REDES FAMILIARES EN LOS VALLES ORIENTALES DE JUJUY (ARGENTINA) ENTRE 1852 y 1910
}

\author{
THE LENGTHS THREADS OF WEFT: ETHNOGRAPHIC NOTES AND ANALYSIS OF FAMILY NETWORKS IN THE \\ VALLEYS EAST OF JUJUY (ARGENTINA) BETWEEN 1852 AND 1910
}

Federico Fernández ${ }^{*}$

antropo428@yahoo.com.ar

\begin{abstract}
RESUMEN: En este texto se analiza el fenómeno del compadrazgo bautismal descripto para un amplio sector en América Latina, a la manera de un dispositivo socio-parental a través del cual es posible visualizar la reproducción de formas vinculares que expresan intercambios simbólicos y jerarquías entre grupos familiares. Los resultados obtenidos tras la pesquisa, muestran que este dispositivo socio-parental, no constituye en sí mismo un mecanismo causal de las relaciones, sino que permite la construcción de un conjunto identificable de tramas relacionales que pueden ser descriptas e interpretadas bajo el denominado análisis estructural de redes.
\end{abstract}

PALAVBAS-ChAVE: Compadrazgo, Redes, Historias familiares.

ABSTRACT: In this text discusses the phenomenon of the baptismal godparents described a wide sector in Latin America, in the manner of a partner-parental device through which it is possible to see the reproduction of forms relational expressing symbolic exchanges and hierarchies between family groups. The results obtained from the research, show that this device, does not constitute in itself a causal mechanism of relations, but allows the construction of an identifiable set of relational patterns that can be described and interpreted under the so-called structural analysis of networks.

KEYWORDS: Godparents, Network, Family stories.

\section{Introducción}

(...) de igual modo que no hay que decir que la copa se ha roto porque una piedra la ha golpeado, sino que se ha roto, cuando la piedra la ha golpeado, porque era rompible, tampoco hay que decir (...) que un acontecimiento histórico ha determinado un comportamiento, sino que ha tenido ese efecto determinante porque un habitus susceptible de ser afectado por ese acontecimiento le ha conferido esa eficacia (BOURDIEU, 1999, p. 196)

La idea central del presente texto es la de re-pensar, a partir de un estudio de caso, los vínculos de apadrinamiento bautismales (parentesco espiritual y/o compadrazgo) descriptos para un vasto sector latinoamericano, a la manera de un dispositivo socioparental a través del cual es posible visualizar la producción y reproducción de formas vinculares que expresan jerarquías de status, cierres y aperturas de relaciones entre grupos familiares. Este dispositivo socio-parental, lejos de constituirse en un mecanismo causal de

\footnotetext{
* Profesor Adjunto de la Cátedra Antropología Social y Cultural en la Universidad Nacional de Jujuy. Investigador Asistente del CONICET. Licenciado en Antropología (FHyCS-UNJu) y Doctor en Ciencias Sociales (Orientación en Historia) por la FFyL de la Universidad Nacional de Tucumán.
} 
las relaciones, ha configurado históricamente y aún persiste en la actualidad, un conjunto identificable de tramas relacionales factibles de ser descriptas y analizadas bajo el denominado análisis estructural de redes.

Es bastante conocida la importancia analítica que conlleva para Pierre Bourdieu el término habitus. Sin embargo, y debido en parte a la complejidad y densidad teórica desarrollada por este mismo autor, es menos conocida la noción de dispositivo que el mismo Bourdieu enfatiza en sus texto cuando refiere a la definición de habitus. En efecto, la idea de dispositivo y/o disposiciones en los análisis de Bourdieu, representa a ese conjunto de acciones y prácticas vinculadas al comportamiento de los agentes no susceptibles de ser asimiladas a la noción de causa-efecto, sino que más bien se trata de acciones que son el producto, y al mimos tiempo reproducen tanto posiciones (relaciones) de esos agentes, como así también los condicionantes que esas relaciones producen en los individuos y grupos sociales históricamente situados.

El padrinazgo bautismal, desarrollado en la península ibérica e impuesto en Latinoamérica a través de instituciones eclesiástica, constituye especialmente en nuestro continente un ejemplo de práctica de alta ritualidad cuya estructura relacional original ha mutado, pero cuyo significado y sentido práctico sigue guiando, es decir, continúa produciendo y reproduciendo determinadas formas elementales de vinculación (económicas, políticas, religiosas, afectivas) entre grupos sociales que habitualmente han ocupado y ocupan posiciones disimiles dentro de la estructura social.

Los apadrinamientos bautismales generan un vínculo específico denominado compadrazgo, el cual constituye una serie de derechos y obligaciones muy variables y no especificadas totalmente de manera escrita entre: padrinos/madrinas, el hijo/hija bautizado (ahijado), y los progenitores del niño o la niña bautizada ${ }^{1}$.

Según los ya clásicos escritos de Nuttini y Bell, el compadrazgo posee una distribución geográfica que abarca: “(...) los Estados Unidos, toda Mesoamérica, el caribe y Sudamérica" (NUTTINI; BELL, 1989, p. 13).

\footnotetext{
${ }^{1}$ Para Nuttini y Bell, los vínculos de compadrazgo en México y en amplios sectores poblacionales de América Latina, a diferencia de la tradición católica occidental, refuerzan fuertemente el lazo entre compadres (padres padrinos, madres - madrinas), con lo cual la niña o niño bautizado ocupa un segundo plano, al menos hasta la llegada de la edad adulta.
} 
Sin embargo, y tal como lo deja entrever Mendoza Ontiveros, el interés de la antropología americanista por este tema en particular, se ha basada no sólo en la dispersión geográfica del fenómeno, sino que también y fundamentalmente, en el análisis operacional de éste tipo de vínculos para establecer trayectos diacrónicos y sincrónicos del cambio social en gran parte de la región (MONTES ONTIVEROS, 2010); y muy especialmente en lo que respecta a las relaciones económicas, políticas y religiosas entre grupos sociales que han fluctuado históricamente entre los espacios rurales y urbanos.

Esto último es particular y explícitamente afirmado por una amplia gama de investigadoras e investigadores vinculados a corrientes funcionalistas y estructuralfuncionalistas, que han llevado a cabo extensas investigaciones especialmente en Centroamérica. Así, por ejemplo, para Nuttini y Bell, el compadrazgo constituye una institución que:

(...) en sus varias formas es significativa funcionalmente en una variedad de situaciones, comprendiendo desde el campesinado comunal, hasta las sociedades urbanas altamente estratificadas. Estructuralmente, el compadrazgo es un mecanismo importante de interacción social que afecta grupos que oscilan en amplitud desde el villorio hasta la nación. (NUTTINI; BELL, 1989, p. 13).

La caracterización del compadrazgo (eje central del parentesco ritual) como una institución que se expresa a través de una serie de mecanismo sociales diversos, ha concitado debates teóricos y metodológicos que aún siguen generando una "divisoria de aguas" entre muchos investigadores.

La pesquisa de Arantes, en su trabajo pionero sobre el compadrazgo en el Brasil rural, define a este tipo de vínculo desde una perspectiva ligada con la noción de estructura ideada originalmente por Levi-Strauss (ARANTES, 2011). A partir de su análisis centrado fundamentalmente en el trabajo empírico llevado a cabo en las comunidades campesinas del estado de Bahia, sugiere entender a la estructura del compadrazgo como:

(...) structure may be defined, then, as a set of size $\mathrm{N}$ of ordered pairs of dyadic relations linking three elements: a sponsored child, parents and godparents. (...) In the case of the godparent/godchild relations, when the sponsored is still young, he reciprocates the godparents' gifts mainly with affection and respect, material responsibilities being transferred to his parents. As he grows up, he will be expected to help the godparent in an increasingly more effective way; then, the basic pattern of the exchanges will depend on relevant aspects of the social structure (class structure, neighborhood, kinship etc), as in the compadres' relation. At the symbolic 
level, both connections are ideally asymmetric, the status of godparent being slightly higher than the others (ARANTES, 2011, p. 95).

Para Lanna, por ejemplo, el compadrazgo en Brasil parece no tener la misma presencia que en el resto de Latinoamérica -lo cual no implica que no tenga importancia- $y$, al contrario de lo que se afirma para el caso Mexicano, los compadrazgos bautismales en Brasil no podrían ser descritos como meros mecanismo de extensión de lazos que se cristalizan en una institución social (LANNA, 2009), sino que más bien, bajo la perspectiva asumida por este autor, constituye, al menos en una de sus aristas simbólicas, un vínculo de intercambio asimétrico de poder donde la persona (niño o niña/ahijado o ahijada) forma parte del intercambio (en términos simbólicos) ofrecido por parte de los progenitores hacia sus compadres. ${ }^{2}$

Los escritos de Mendoza Ontiveros sobre los yaqui de Sonora (México) y Arizona (EEUU), también tiene como punto de partida teórico la noción estructural planteada por Levi-Strauss especialmente en su Antropología Estructural (MENDOZA ONTIVEROS, 2004). Sin embargo, y a diferencia de lo planteado por los autores anteriores, Mendoza Ontiveros define al compadrazgo como una cadena lógica subyacente entre significados y significantes en donde las diadas y/o triadas son sólo las unidades empíricas observables. En este sentido, su planteo establece que resulta insuficiente la descripción empírica y funcional de todos los tipos de compadrazgos que comprenden al parentesco ritual de una comunidad o poblado. Resulta necesario, además de las descripciones etnográficas, pensar la información empírica en términos teóricos, de tal modo que se pueda reconocer aquello que subyace a todos los vínculos registrados bajo la órbita del parentesco ritual.

En términos esquemáticos, se puede establecer entonces dos grandes bloques teóricos a través de los cuales la antropología social en Latinoamérica ha analizada los vínculos de compadrazgo generados a través del principal ritual sacramentales para los católicos como lo es el bautismo. Por un lado aquellos estudios centrados fuertemente en la descripción y el análisis funcional e ideológico del compadrazgo. Por otro lado, el análisis de corte estructuralista, enfocado más bien en los contenidos subyacentes de la vida social, a través del cual se intenta comprender el fenómeno del compadrazgo a la manera de un

\footnotetext{
${ }^{2}$ Resulta necesario aclarar aquí que para Lanna el enfoque de Arantes, si bien constituye una perspectiva que excede la visión funcional del compadrazgo, no logra superar la noción de las relaciones de compadrazgo en términos instrumentales.
} 
cadena de unidades relacionales, cuyo valor simbólico inconsciente es necesario desentrañar en términos analíticos.

Como se ha señalado al inicio del presente texto, la idea de comprender este fenómeno tan extendido en América Latina a la manera de un dispositivo socio-parental expresado a través de una red, tiene como pretensión la posibilidad de conjugar las líneas rectoras de ambos modelos teóricos. Así pues, tomando la carga teórica que Bourdieu señala en torno a la idea de disposiciones, es posible y necesario no sólo el análisis sincrónico contemporáneo, es decir, etnográfico. Sino que además resulta imprescindible un análisis histórico situado del fenómeno como tal, no ya con la pretensión de describir la funcionalidad del compadrazgo bajo determinados periodos espacio-temporales, sino más bien con el objeto de registrar las regularidades estructurales del compadrazgo como actividad ritual sacralizada y/o secular.

Desde esta perspectiva, en las líneas que siguen se dará cuenta de una investigación iniciada en la contemporaneidad etnográfica, pero que ha devenido en una serie de preguntas con respuestas cuya exégesis se encuentra determinada por el análisis del tiempo pasado. Sin dudas que los fenómenos del compadrazgo tal y como se presentarán a continuación poseen mayores similitudes de análisis con el área geográfica más cercana a la unidad de estudio, es decir, a los registros etnográficos y etnohistóricos relevados para el área sur-andina. Sin embargo, y debido a que la intención de este trabajo es el de mostrar un panorama más general de un fenómeno complejo y ampliamente extendido, he optado por citar trabajos elaborados en base a poblados y grupos socio-étnicos que se encuentran por afuera de la región andina tradicional, como los casos citados para Brasil y México.

\section{Principales características estructurales de la población vallegrandina}

La unidad de análisis de la presente pesquisa comprende la extensión políticoadministrativa del denominado departamento Valle Grande, el cual se encuentra ubicado en el extremo oriental de la Provincia de Jujuy (territorio fronterizo en sus límites norte y noroeste con los países de Bolivia y Chile).

La población que ha residido históricamente, y reside actualmente dentro del área Valle Grande, presenta niveles de variación menores entre el primer registro censal nacional 
del siglo XX realizado en 1914 donde el número de habitantes en todo el área era de 1721 pobladores entre hombres y mujeres, y el último censo nacional del siglo XX realizado en el año 1991, donde la población registrada para Valle Grande fue de 1978 habitantes. ${ }^{3}$

En términos generales, todo el territorio que comprende el actual departamento Valle Grande puede ser dividido, tomando como referencia los espacios eco-ambientales y los metros de altura sobre el nivel del mar, en dos grandes regiones. Una región de altura ubicada entre los 2500 metros y por arriba de los 3500 metros sobre el nivel del mar (zona alta de Valle Grande), y otra micro-región dentro del mismo límite departamental, que se encuentra por debajo de los 2500 metros (zona baja de Valle Grande). ${ }^{4}$

Las determinaciones geográficas y eco-ambientales de toda esta región, constituyen una información fundamental para comprender las relaciones entre la dinámica poblacional local-regional dentro de una duración histórica de corte alcance (aproximadamente desde mediados del siglo XIX hasta la segunda mitad del siglo XX), las bases económicas dominantes en todo el área, y los dispositivos socio-parentales más elementes desarrollados durante este periodo temporal.

Tal como lo ha desarrollado Teruel, la propiedad de la tierra de carácter latifundista pasada la mitad del siglo XIX en la provincia de Jujuy, incluyó al menos tres grandes porciones de tierras productivas (TERUEL, 2006). Una de estas grandes extensiones, la correspondiente a los valles subtropicales, incluía al departamento Valle Grande que por aquellos años:

(...) estaba dominado por la propiedad de la familia Valle (Caspalá, Santa Ana y Valle Grande). Durante el mandato de Tello [Gobernador de la provincia] se intentó regularizar la situación de Santa Bárbara, y lo más significativo, en 1887, se subdividió las tierras de Valle Grande, posibilitando a los campesinos que la arrendaban, el acceso a la propiedad de sus parcelas (TERUEL, 2006, p. 314)

La situación de compra de tierras con derechos de propiedad por parte de los campesinos-indígenas del valle en 1887, tuvo que haber impactado profundamente en todos

\footnotetext{
${ }^{3}$ Los últimos datos censales (2001 y 2010) para Valle Grande expresan una cantidad de población variable que ronda entre los 2300 y 2600 habitantes.

${ }^{4}$ Existen actualmente un total de 10 parajes rurales, dos de ellos constituyen cabeceras administrativas de toda el área (Valle Grande y Santa Ana). Esta decena de pequeños conglomerados rurales se encuentran distribuidos alrededor de una zona montañosa que combina la vegetación y el clima de las yungas o selva de montaña, con el cordón montañoso andino.
} 
los niveles organizativos de la vida social en la región debido fundamentalmente a dos razones concretas: 1- Al ser la ganadería (principalmente ganado vacuno y en menor medida ovino), y la agricultura de maíz junto a una variedad de tubérculos la base económica principal del área; el desplazamiento de ganaderos y agricultores con sus animales a lo largo de todo el valle combinando diferentes estaciones climáticas y ecológicas de acuerdo al mosaico natural de los pisos de altura, generó una práctica social y cultural de larga tradición como lo es, incluso en la actualidad, la trashumancia cíclica. 2- Al dividirse gran parte del departamento en estos nuevos grupos familiares de propietarios (anteriormente arrendatarios), esta dinámica de movilidad, en la cual se incluye el traslado, más el cuidado semipermanente de los animales y los sembradíos ubicados mayormente fuera de los poblados rurales, tuvo que haber generado una mayor extensión de lazos familiares bajo la búsqueda de fragmentos de tierras y residencias semipermanentes bilocalizadas (tanto en la zona alta, como en la zona baja del valle).

Producto en gran medida de estas condiciones estructurales, los vínculos de parentesco y la organización social predominante entre los vallegrandinos en el presente etnográfico, resultó ser la familia extendida con residencia múltiple ${ }^{5}$. Esta forma particular de organizar los vínculos parentales, ha sido y es la encargada de establecer las pautas organizativas de mayor relevancia en el espacio público como lo son el traslado y manutención de animales, el ritual de la marcada de ganado, organización de bautismos, casamientos, fiestas de carnaval, eventos religiosos de importante envergadura; como así también, en la mayoría de los casos, la familia extensa se encarga de "guiar" las decisiones económicas intra-familiares que han generado la búsqueda de trabajos asalariados temporarios por parte de integrantes en edad activa que crecieron en los grupos familiares de origen vallisto.

Una descripción rápida y poco profunda de la organización social de estas poblaciones rurales en la contemporaneidad, dará como resultado una imagen de armonía campesina hogareña, en donde cada una de las viviendas, en su mayoría pequeñas de tamaño, albergan sólo a la familia nuclear, más un pequeño patio con plantas y animales domésticos. Sin embargo, y a medida que se avanza en el conocimiento de las dinámicas

\footnotetext{
${ }^{5}$ La familia extensa posee una composición variable en el número de integrantes e incluye a más de dos generaciones que comparten una o más residencias. Se diferencia de la denominada familia nuclear en tanto que a esta última la componen únicamente un par de progenitores y su descendencia.
} 
socio-parentales y espaciales de los grupos familiares que habitan el valle, el cuadro se complejiza bastante en tanto la familia nuclear, en la práctica, sólo convive bajo un mismo techo en contadas excepciones a lo largo del año. Así, por ejemplo, durante la época de invierno y verano, la gran mayoría de hombres y mujeres se encuentran cuidando de sus animales, viviendo por semanas en puestos de pastaje lejos del pueblo, o en la ciudad, residiendo temporariamente junto a algún pariente para poder realizar trámites administrativos. En suma, la familiar nuclear en pocas ocasiones logra estar junta dentro de una misma residencia.

De acuerdo a las investigaciones históricas y antropológicas desarrolladas en el área (FERREIRO; FERNÁNDEZ 2013; FERNÁNDEZ, 2015), toda esta región se caracteriza por una marcada endogamia regional en las uniones conyugales. Esta pauta se expresa en una serie amplia de documentos bautismales y actas matrimoniales correspondientes a Valle Grande, en donde se ha podido consignar un alto número de hijos de mujeres y hombres cuyos padres y abuelos se han unido matrimonialmente con parejas del mismo poblado y/o en poblados distintos pero dentro de la misma región a lo largo de las dos últimas centurias.

En lo que respecta específicamente al registro de procedencia de los padrinos/madrinas bautismales de niños y/o niñas nacidas en Valle Grande, es posible reconocer cambios en el origen tanto de padrinos y madrinas. Este cambio se visualiza de manera progresiva en una serie temporal que tiene como punto de despegue pasada la segunda mitad del siglo XIX, y cuya expresión numérica refleja una mayor cantidad de apadrinamientos de bautismos a cargo de hombres y mujeres foráneas al área Valle Grande.

En trabajo anterior, se ha desarrollado la hipótesis de que este cambio en lo referente al crecimiento de padrinos/madrinas exógenos a Valle Grande, podría haberse debido, en parte, a la necesidad de los campesinos del valle de vincularse con personas de confianza -valor fundamental para todo compadrazgo-, lo cual les habrían facilitado el acceso al dinero necesario para comprar tierras en el año 1887.

De acuerdo con los registros etnográficos realizados entre los años 2008 y 2013, para un número significativo de vallegrandinas y vallegrandinos residentes y no residentes en la región, e independientemente de un rango etario determinado, existe una importancia decisiva en lo que respecta al conocimiento detallado de los vínculos parentales y de compadrazgo. Durante una de mis primeras estadías de trabajo de campo, he podido 
constatar esto a través de la preocupación de Laureano ${ }^{6}$, un joven que por el año 2008 tenía unos 20 años edad. En aquella ocasión Laureano se encontraba visiblemente preocupado por "encontrar" una compañera conyugal que sea del mismo pueblo de Santa Ana (zona alta del departamento), pero que al mismo tiempo no este emparentada con el grupo familiar de su madre, ni de su padre, esto es, en la medida de lo posible, una mujer que no lleve el mismo apellido de sus padres por ambas líneas (paterna y materna).

Durante la temporada invernal del año 2009, mientras todavía me encontraba recabando información genealógica e historias familiares del poblado de Santa Ana, tuve una charla informal con María, una mujer de más de cincuenta años de edad, que en aquel momento residía en el sector alto del poblado de Santa Ana. La breve historia relatada por María consistió en lo siguiente:

Aquí cerquita nomas, en la casa de la Florencia, he visto un hombre con poncho, no recuerdo bien quien era ... iba en caballo, daba vuelta la casa por afuera nomas ... Era oscuro pero madrugada ya, perros ladrando, ladrando fuerte y de ahí se fue ... No era fantasma ... ¿qque será pues?

Tras largo tiempo no he logrado comprender la relación entre el relato de María, en apariencia una leyenda local, y mis preguntas de investigación centradas más bien las alianzas parentales y las historias familiares. Por el contrario, las preocupaciones evidentes de Laureano eran del todo claras y "encajaban" bastante bien con lo observado y registrado en mis largas estadías en Santa Ana; pero María, ¿a qué se refería con aquel relato del hombre con poncho y a caballo?, ¿cómo se relacionaba esto con las historias familiares locales?

Trece años después de haber registrado aquella historia, y tras haber leído la compilación de relatos populares del norte argentino analizados por Rubinelli bajo el título: Entre condenados, ucumares y gualichadados: relatos andinos tradicionales, he podido repensar el breve relato que María contó en Santa Ana.

Los condenados, según lo analiza Rubinelli, representan la figura encarnada de individuos que en vida han cometido una serie de transgresiones por las cuales deben "pagar", permaneciendo en un espacio-tiempo liminal entre la vida y la muerte, hasta tanto sufran por las fechorías realizadas en el mundo de los vivos, es decir, en el presente socio-

\footnotetext{
${ }^{6}$ Para preservar la identidad de las personas con quienes construí la información etnográfica, los nombres personales expresados en el presente texto son ficticios.
} 
cultural de la comunidad en donde han desarrollado su ciclo vital (RUBINELLI, 2016). Entre las transgresiones en vida por las cuales una persona puede transformarse en condenada se encuentran las relaciones sexuales entre parientes consanguíneos, en especial padre/hija, madre/hijo, pero también esta prohibición es extensiva a los vínculos comadre-compadre, compadre-comadre y padrinos/madrinas para con ahijadas/ahijados. Asimismo, el tabú que da nacimiento a los condenados, no sólo abarca a los moradores del poblado, sino que también puede afectar a sacerdotes en caso de que estos transgredan los límites establecidos por la misma comunidad.

En los relatos analizados en el libro de Rubinelli, los condenados aparecen siempre de noche, en muchos casos con figuras claramente humanas y son referidos como desconocidos de la ciudad o vecinos de pueblos cercanos. También en muchos casos son los perros los que avisan la presencia de un condenado, quienes por lo general, cuando llega la noche, se encuentran merodeando las casas del poblado en la búsqueda de comida.

Bajo estas narrativas construidas y transmitidas oralmente entre gran parte de los sectores populares rurales y urbanos del norte argentino, el relato de María parece cobrar un sentido más específico. Se trata, en suma, del tabú del incesto como norma -tal y como lo ha analizado Levi-Strauss-, pero que en este caso se hace extensivo y abarca a las relaciones de compadrazgo, en especial aquellas generadas a través del bautismo, e incluso llega, por intermedio de un complejo sincretismo religioso, a la figura del sacerdote cristiano (autoridad máxima en el primero de los actos sacramentales).

La prohibición de mantener relaciones sexuales entre todos los que participan de las relaciones de compadrazgo a partir del bautismo, constituye una proscripción bastante extendida en toda América Latina. El caso particular y bien documentado de los yaqui en el norte de México ${ }^{7}$, o la presencia de la figura de los condenados en los relatos de gran parte

\footnotetext{
7 Según Mendoza Ontiveros, los yaqui presentan un sistema bastante singular y extensivo de compadrazgo. Dentro de este grupo étnico, existen un número importante de padrinos en tanto que éstos son solicitados para acompañar a los iniciados en todos los ritos de paso vinculados a los ciclos vitales, y que son fuertemente prescriptos por la comunidad. El bautismo, uno de los principales ritos practicados por los yaqui, constituye no sólo un poderoso ejemplo de sincretismo religioso, sino que además se presenta en este caso bajo una pauta precisa: los padrinos y madrinas no pueden tener, ni haber tenido relaciones maritales entre sí (MENDOZA ONTIVEROS, 2004). Esto es, en otras palabras, la extensión del tabú del incesto que rige las relaciones entre parientes considerados como consanguíneos abarca aquí a los apadrinadores bautismales.
} 
de los sectores populares de Bolivia y Perú (SPEDING, 2011), dan cuenta de este tabú del incesto extendido hacia los vínculos contraídos por parentesco espiritual.

La importancia del tabú del incesto en la perspectiva de Levi-Strauss resulta crucial para comprender los límites que suponen los vínculos endogámicos y exogámicos entre grupos sociales. En este sentido, y teniendo en cuenta lo expresado hasta aquí, puede suponerse que, al menos en el presente etnográfico local y regional, el carácter extensivo que posee el tabú del incesto incluyendo fundamentalmente a los vínculos de compadrazgo sacramental, tuvo que haberse gestado a la manera de un dispositivo socio-parental bajo condiciones estructurales e históricas particulares dentro de la región. Bajo esta premisa, en los siguientes apartados se intentará responder a una incógnita que considero central: ¿Qué posición, en términos relacionales, ocuparon aquellos individuos (hombres y mujeres) registrados con el mismo apellido entre mediados del siglo XIX y principio del XX, y cuál fue el lugar de los padrinos y madrinas bautismales en estos esquemas familiares?

\section{Ordenamiento de fuentes y análisis socio-histórico de redes familiares en Valle Grande}

La descripción y análisis que se desarrolla a continuación se basó en la búsqueda, selección y ordenamiento reticular ${ }^{8}$ (MOLINA, 2000) de tres tipos de fuentes históricas distintas: un padrón censal de 1852, libros de actas bautismales correspondientes al periodo 1850-1910, y los registros de pago sobre propiedades territoriales efectuados por pobladores locales en el año 1887.

Todo indica que quienes llevaron a cabo el registro censal de 1852 tuvieron como criterio de delimitación la unidad residencial de los censados (el hogar). Así pues, los listados del censo se inician con un cabeza de hogar, generalmente un varón adulto, le sigue inmediatamente una mujer adulta portadora de un apellido por lo general distinto a quien encabeza el listado, y continua con el nombre y apellido de individuos de igual y/o diferente sexo portadores del mismo apellido de quien figura como cabeza de padrón. Existen, además de la secuencia señalada de nombres y apellidos, individuos (varones y mujeres) que

\footnotetext{
${ }^{8}$ El ordenamiento y análisis reticular, junto con las interpretaciones topológicas de las redes, constituyen los dos pilares fundamentales del análisis formal de redes sociales descriptos por Molina. El ordenamiento reticular de los datos refiere a la construcción de matrices cuadradas con datos binarios. Bajo la lógica del programa informático UCINET.6 utilizado en esta investigación, las matrices reticulares permitieron establecer cálculos numéricos, mientras que los gráficos de redes, como se verá más adelante en el texto, habilitan la interpretación topológica de la información previamente seleccionada y ordenada en matrices reticulares.
} 
no poseen el apellido del varón con el cual se inicia el listado, ni tampoco tienen el apellido de la mujer que figura inmediatamente a continuación del cabeza de padrón. Estas personas han sido consignadas en el presente estudio como: Agregados de la unidad doméstica.

La información originada a partir del censo de 1852 ha sido completada con los datos provistos por las actas bautismales, y el registro de compra-venta de tierras correspondiente al año 1887. De este modo, se partió de un conjunto delimitado de personas registradas en el censo, para luego realizar el "rastreo", a través de la información eclesiástica, del lugar de nacimiento consignado de estos mismos individuos, la edad, el lugar de residencia y procedencia de los padres, y el lugar de residencia y procedencia de los padrinos/madrinas bautismales. Como se verá en detalle, en dos casos puntuales se han podido llegar a identificar los vínculos de parentesco consanguíneo y político, nombre y apellido de los compadres, el lugar de procedencia, residencia, y el monto pagado en 1887 por la persona adultas señaladas en las actas bautismales.

Todo este conjunto de datos han sido ordenados a partir de un Ego varón que figura como cabeza de padrón en el mencionado registro censal de 1852. En este texto en particular, se ha limitado el análisis a la reconstrucción de dos grupos de familias. La primera de ellas es encabezada por Fermín Castañeda, quien, según lo deja entrever la información histórica disponible, representó a una de las familias de mayor importancia desde mediados del siglo XIX (FERREIRO, 2016) para todo el valle ${ }^{9}$. El segundo grupo familiar lleva como primer nombre y apellido de padrón a Justo Cruz, principal referente del conglomerado familiar de mayor importancia en toda la zona alta de la región vallegrandina.

La codificación de los datos utilizados ha sido la siguiente: se consignaron los nombres y apellidos de los individuos que figuran en las diferentes fuentes (censo, bautismos, registros de propiedad), seguido del lugar de procedencia y/o residencia escrito por el censista y el sacerdote a cargo del acta bautismal. Ej: Fermín Castañeda (Valle Grande), Ceferina Cáceres (Humahuaca), Bonifacio Lamas (Humahuaca).

\footnotetext{
${ }^{9}$ El análisis genealógico y de redes sociales desarrollado por Ferreiro, da cuenta de la importancia crucial de la figura de Fermín Castañeda para este periodo. Castañeda residió primero en la parte alta del valle (poblado de Caspalá), luego se asentó junto a su familia en el poblado de Valle Grande (zona baja), fue el encargado de confeccionar el padrón censal local de 1852, también ejerció como policía a cargo de toda la región, fue comerciante, y un solicitado padrino de bautismo por los vallegrandinos de la época.
} 
Esta información ya codificada fue ordenada dentro de un cuadro de doble entrada conocido como matriz cuadrada o reticular en el lenguaje formal de redes. En el caso particular de la presente investigación, las dos matrices confeccionadas son cuadradas y de carácter simétrico en relación a la dirección de los vínculos.

Por intermedio del generador de gráfico de redes (NetDraw) incluido en el paquete informático UCINET. 6, se construyeron los gráficos de redes que se visualizan más abajo en el texto. La base de dato sobre la cual se generaron los gráficos, fueron las matrices cuadradas simétricas.

Las referencia de colores exhibidas en los gráficos de redes señalan las siguientes características: Color rojo: Ego masculino portador del apellido transmitido. Color marrón: vínculos por alianza. Color negro: integrantes de la unidad doméstica que no comparten el mismo apellido del Ego masculino. Color azul: Siblins (hermanos del mismo y/o diferente sexo). Color verde: padrinos y/o madrinas de bautismo.

Los diferentes diámetros en las formas de los nodos, señalan el mayor y/o menor rango de centralidad que presentan los vértices. Se siguió aquí el cálculo de centralidad de grado descripto por Hanneman, donde se computan la cantidad de lazos (aristas) que recibe y/o emite un nodo (HANNEMAN, 2001). En la presente pesquisa, este rango ha sido medido teniendo en cuenta la cantidad de vínculos familiares directos entre los nodos, por ejemplo, se partió del nodo Ego masculino cabeza de padrón, y se contabilizó cada uno de sus vínculos directos (hijos-esposa-compadrazgos). Asimismo, se estableció el rango de intermediación, es decir, la cantidad de nodos intermedios que separan a un nodo de otros (HANNEMAN, 2001). Este último cálculo, a diferencia del rango de centralidad, no ha sido expresado en el diámetro de los vértices de la red dado que complejizaba su visualización; aunque sí se mencionan los nombres que componen las medidas de centralidad e intermediación de las redes graficadas. 


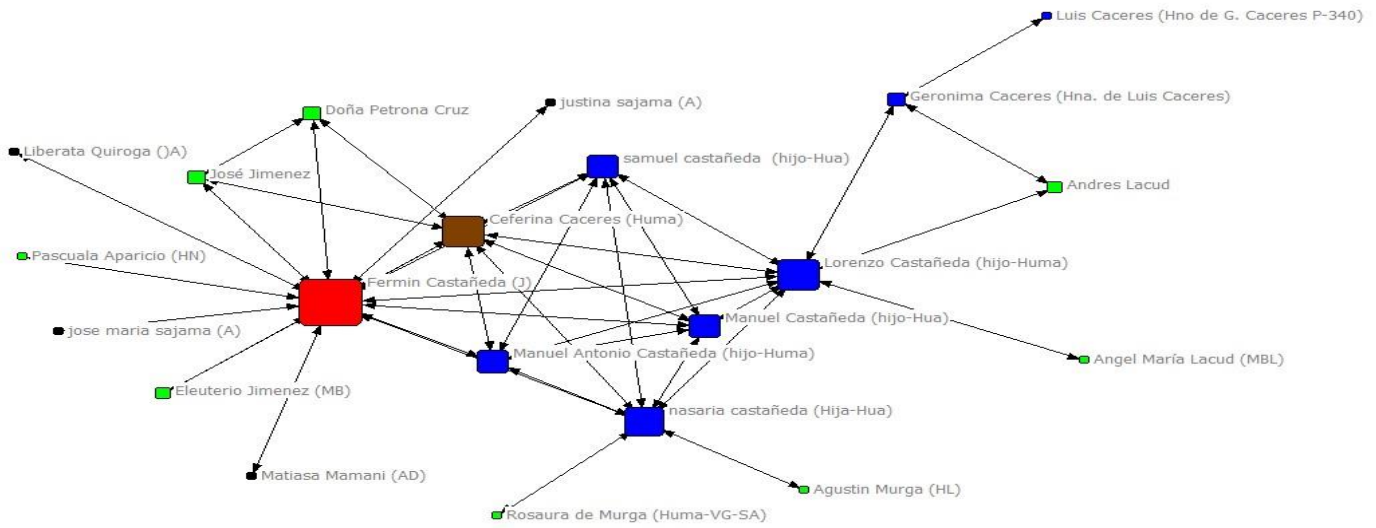

Gráfico I. Mapa de redes parentales y lugares de residencia de la familia Castañeda (zona baja de Valle Grande)

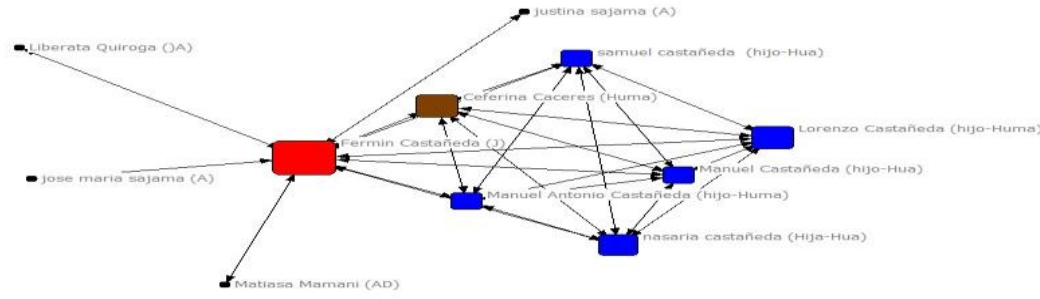

Grafico IA. Mapa de redes parentales y lugares de residencia de la familia Castañeda sin la presencia de padrinos y/o madrinas de bautismo

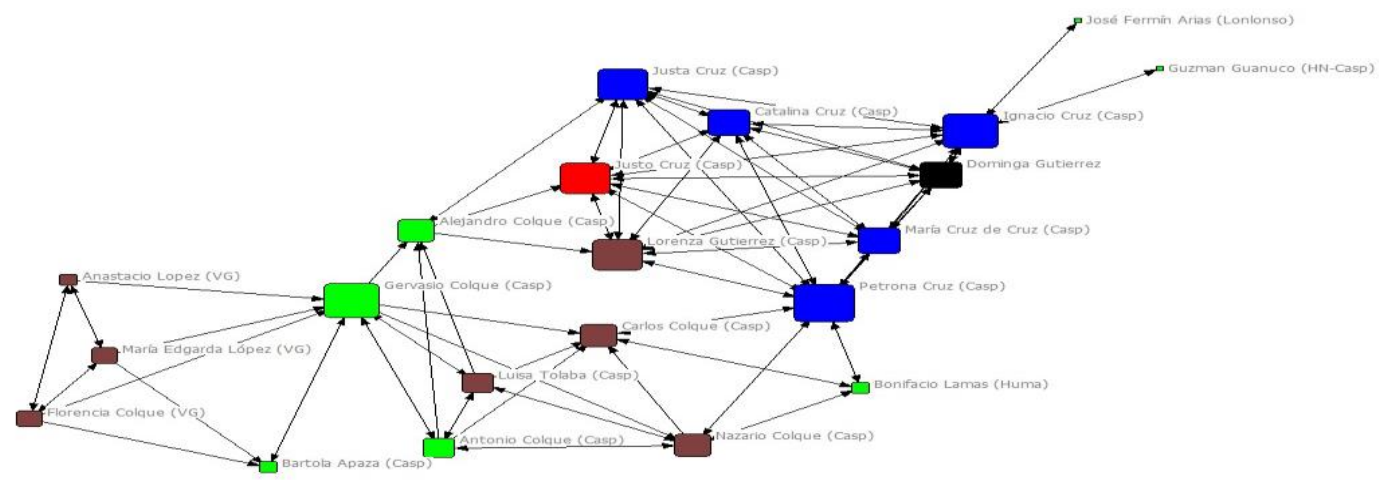

Gráfico II. Mapa de redes parentales y lugares de residencia de la familia Cruz (zona alta de Valle Grande) 


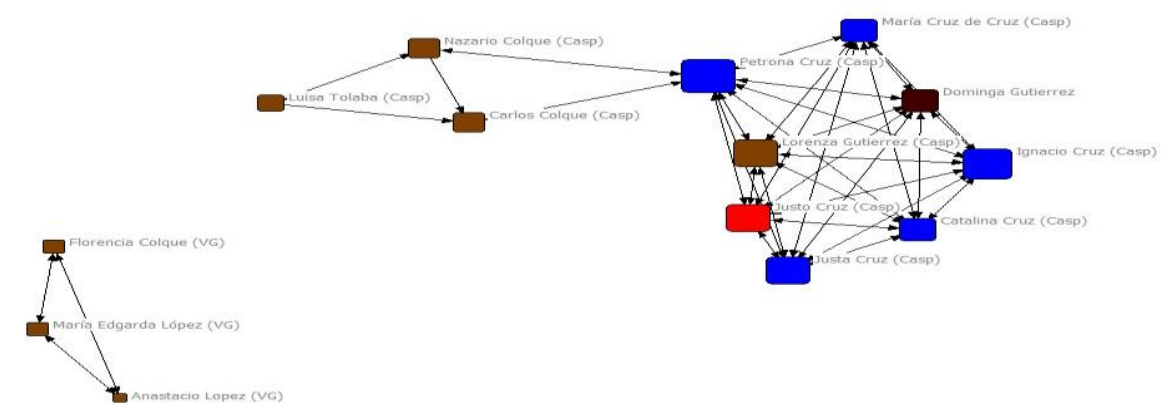

Gráfico IIA. Mapa de redes parentales y lugares de residencia de la familia Cruz sin la presencia de padrinos y/o madrinas de bautismo

\section{Análisis e interpretación de los datos}

El mapa de redes $\mathrm{N}^{\circ}$ I nos muestra un entramado relacional cuya mayor densidad se encuentra entre los nodos que representan a Fermín Castañeda (ego masculino), su mujer Ceferina Cáceres, los hijos de ambos (Ceferina, Lorenzo, Nazaria, Marcelo, Samuel y Manuel).

Si se observa detenidamente la forma gráfica que presenta esta red, se cae en cuenta que la densidad del centro se va disolviendo hacia los laterales a través de vínculos entre hermanos, que a su vez se relacionan con lazos de padrinazgos hacia afuera del centro. Naturalmente, en la medida en que nos alejamos del núcleo de la red, los nodos van reduciéndose en tamaño, puesto que los vínculos directos recibidos y emitidos por los vértices son cada vez menos. Sin embargo, resulta significativa la comparación entre los lugares que ocupan los individuos en los cálculos de centralidad e intermediación.

Geronima Cáceres ocupa el lugar $N^{\circ} 9$ en el cálculo de centralidad, mientras que el mismo nodo ocupa el puesto $\mathrm{N}^{\circ} 4$ en la tabla de intermediación. Este cambio de posición en el rango de la red de Geronima Cáceres, se debe al lugar que ocupa dentro de la red. Si bien es un nodo con pocos vínculos directos, constituyó un nexo clave para conectar el centro del gráfico de la red con la periferia lateral. Así púes, nótese que Gerónima Cáceres no formó parte de la familia Castañeda de manera directa, es decir, a través de la descendencia del matrimonio Castañeda-Cáceres, sino que su vínculo para con el núcleo de la red es a través de Lorenzo Castañeda (hijo de Fermín Castañeda y Ceferina Cáceres), a partir de un vínculo de compadrazgo entre Lorenzo y Gerónima originado por el apadrinamiento de Lorenzo 
Castañeda para con Ángel Lacud (hijo de Andrés Lacud y Geronima Cáceres, ambos registrados en el poblado de Valle Grande).

El mapeo de redes IA expresa con mayor claridad el papel de intermediario y/o nexo que ocupó Geronima. Al extraer de la red los lazos de compadrazgo, Lorenzo Castañeda queda desvinculado de Geronima, la familia Lacud y fundamentalmente no llega a conectarse con Luis Cáceres (hermano de Geronima), quien figura como uno de los compradores de terrenos que más dinero aportó -un total de 340 pesos-, para la compra de fincas en 1887 dentro de la región vallegrandina.

La diversidad en lo que respecta a la procedencia de quienes componen este primer grupo familiar, constituye un buen ejemplo de la movilidad poblacional y la consecuente residencia múltiple ocupada a lo largo del tiempo por los pobladores vallistos. Así, por ejemplo, dentro del grupo familiar de Fermín Casteñeda se encuentran consignados tres poblados diferentes entre sí: el pueblo de Valle Grande, Humahuaca y la localidad de Huacalera (los dos últimos poblados son localidades exógenas al área Valle Grande).

El mapeo de redes número II, refiere a un fragmento de la historia parental de la familia Cruz, apellido mayoritariamente localizado en la región de altura, ubicado hacia el norte de la actual región vallegrandina. A diferencia de lo descripto para el caso de la familia Castañeda, el nodo con mayor centralidad en este segundo mapeo de redes corresponde a una mujer llamada Petrona Cruz. Se trata de una de las hijas mayores de Justo Cruz de Caspala (cabeza de padrón en el censo de 1852). El rango de centralidad alto de Petrona Cruz obedece a que, además de haber tenido vínculos dentro de su familia paterna, estableció una relación directa de apadrinamiento con Bonifacio Lamas (nodo de color verde) quien residía en la vecina localidad de Humahuaca.

En términos generales, la red familiar iniciada por Justo Cruz presenta al menos dos bloque vinculares expresados gráficamente. Uno de estos bloques se encuentra compuesto fundamentalmente por hermanos y hermanas de apellido Cruz (nodos azules), que a su vez han establecidos contactos de padrinazgos (nodos de color verde) referenciados hacia los márgenes de la red. Un segundo bloque relacional incluye a los nodos relacionados a través de las uniones conyugales establecidas por Petrona Cruz, quien además de poseer el más alto rango de centralidad, es también el nodo con mayor nivel de intermediación de toda 
esta red. Esto se debe, como se evidencia en un costado de la red, al hecho de que sus relaciones llegan más allá de sus lazos estrictamente consanguíneos.

En el gráfico de redes parentales y lugares de residencia de la familia Cruz sin la presencia de padrinos y/o madrinas de bautismo, es posible observar de manera precisa la importancia de Petrona Cruz para con el segundo bloque vincular, aquel que incluye los nodos relacionados con uniones conyugales, y que en este caso en particular aglutinan a los apellidos Colque y Tolaba.

\section{Conclusiones}

El resultado del mapeo relacional y territorial presentado, permite representar una dinámica familiar que fue más allá de la residencia micro-local. A través de estas redes parentales (consanguíneas y de compadrazgo), la imagen que predomina es más bien la de una serie de constelaciones concentradas en algunos puntos, pero también dispersas a lo largo y ancho del espacio regional interno y externo a la región vallegrandina.

La noción metafórica que dio origen al título del trabajo: "los hilos largos de la trama", se corresponde con las líneas que conectan a familiares consignados dentro de una misma residencia en 1852, pero también a vínculos de compadrazgo que llegan hasta 1910 y que permiten la conectividad hacia los márgenes extremos de los gráficos. De este modo, la reconstrucción esquemática de dos principales familias, permite visualizar con claridad la importancia posicional de los padrinos y madrinas bautismales.

Como ya se ha señalado, los registros etnográficos -no sólo en el norte de argentina sino también en gran parte de América Latina-, ofrecen una diversidad de pautas sociales en donde se asocian los lazos de compadrazgo con funciones institucionales que estaría expresando, en términos sólo ideológicos para las tradiciones funcionalistas, el cambio social entre la interface rural-urbano, articulando de este modo la transformaciones de grupos parentales "cerrados" hacia las aperturas de nuevos lazos, los cuales exceden el parentesco consanguíneo para insertarse en otra lógica, la de los lazos de compadrazgo.

Para las tradiciones teóricas de corte estructuralista, en cambio, el compadrazgo al igual que las lógicas de parentesco, constituyen unidades significantes que subyacen a la estructura socio-cultural visible empíricamente, y el valor analítico de estas relaciones se 
encuentra en las normas de intercambio; de allí el énfasis de Levi-Strauss en el tabú del incesto como pauta que regula los pares endogamia/exogamia.

Tomando como punto de partida esta última perspectiva, los datos etnográficos e históricos sobre el compadrazgo en Valle Grande, reflejan, al igual que en toda Latinoamérica, la extensión del tabú del incesto intra-familiar hacia los compadrazgo originados en el bautismo. Tal extensión se expresa en la contemporaneidad vallegrandina a través de las transgresiones incestuosas desarrolladas en vida dentro de la comunidad, y por las cuales las personas deben "pagar", tras la muerte, encarnando en la figura del condenado. Sin embargo, este tipo relatos populares, no son entendidos aquí como producto de causalidades históricas o mecanismos institucionales generados de manera consiente por agentes sociales a lo largo del tiempo-espacio histórico local/regional, sino más bien como disposiciones socio-parentales, generalmente inconscientes, que bajo determinadas constricciones históricas, económicas y religiosas, habilitan las formas de intercambio asimétrico entre grupos familiares que ocuparon y ocupan posiciones sociales disimiles en la estructura social local.

\section{REFERENCIAS BIBLIOGRÁFICAS}

ARANTES, A. Compadrio In Rural Brazil. Structural Analysis of a Ritual Institution. Vibrant, Virtual Braz. Anthr. vol. 8, n. 2, Brasília July/Dec. 2011. http://dx.doi.org/10.1590/S180943412011000200005.

NUTINI, H; BELL, B. Parentesco Ritual. Estructura y evolución histórica del sistema de compadrazgo en el Tlaxcala rural. México, D F: Editorial Fondo de Cultura Económico, 1989.

BOURDIEU, P. Meditaciones pascalianas. Barcelona: Editorial Anagrama, 1999.

MOLINA, J. El análisis de Redes Sociales. Barcelona: Bellaterra, 2000.

BORGATTI, S. P.; EVERETT, M. G.; FREEMAN, L. C. Ucinet for Windows: Software for Social Network Analysis. Harvard, MA: Analytic Technologies, 2002-2007.

FERREIRO, J. P; FERNÁNDEZ, F. Nupcialidad, compadrazgo y endogamia en las Yungas de Jujuy (Noroeste de Argentina) durante la primera mitad del siglo XIX. Caravelle. Cahiers du monde hispanique et luso-brésilien, n. 101, 2013, p. 21-56.

FERNANDEZ, F. Redes de apadrinamiento en el oriente jujeño. Periodo 1902-1930. Revista de Estudios Sociales Contemporáneos. IMESC-IDHESI, Conicet, Universidad Nacional de Cuyo, n. 12, 2015, p. 34-48. 
FERREIRO, J P. De barro somos ... Ciclos familiares y genealogía en el poblamiento del oriente jujeño en el siglo XIX. Surandino Monográfico, n. 1, 2016, p. 24-45.

HANNEMAN, R. Centralidad y Poder. In: Introducción a los métodos del análisis de redes sociales. Departamento de Sociología de la Universidad de California. Obtenido el 16 de julio de 2010. En: http://wizard.ucr.edu/ rhannema/networks/text/textindex.html

LANNA, M. A estrutura sacrificial do compadrio: uma ontología da desigualdade? Ciencias Sociais Unisinos. Universidade do Vale do Rio dos Sinos Brasil, vol. 45, n. 2, enero-abril, 2009, p. 5-15.

MENDOZA ONTIVEROS, M. El compadrazgo desde la perspectiva antropológica. Alteridades, vol. 40, n. 20, 2010, p. 141-147.

MENDOZA ONTIVEROS, M. Análisis estructural del compadrazgo yaqui. Ciencia Ergo Sum. Universidad Autónoma del Estado de México, vol.10, n. 3, 2004, p. 259-269.

TERUEL, A. Panorama económico y socio-demográfico en la larga duración. In: TERUEL, Ana; LAGOS, Marcelo (directores). Jujuy en la Historia. De la colonia al siglo XX. Jujuy: EdiUnju, 2006.

LÉVI-STRAUSS, C. Antropología Estructural. Mito, sociedad, humanidades. Buenos Aires: Siglo XXI editores, 2004.

RUBINELLI, M. Entre condenados, ucumares y gualichados. Relatos andinos tradicionales. Jujuy: EdiUnju, 2016.

SPEDDING, A. Sueños, kharisiris y curanderos. Dinámicas sociales de las creencias en los Andes contemporáneos. La Paz, Bolivia: Editorial Mama Huaco, 2011. 